\title{
ON WEAKLY MULTIPLICATIVE INVERSE TRANSVERSALS
}

\author{
by T. S. BLYTH* and M. H. ALMEIDA SANTOS* $\dagger$
}

(Received 3rd July 1992)

\begin{abstract}
We show that an inverse transversal of a regular semigroup is multiplicative if and only if it is both weakly multiplicative and a quasi-ideal. Examples of quasi-ideal inverse transversals that are not multiplicative are known. Here we give an example of a weakly multiplicative inverse transversal that is not multiplicative. An interesting feature of this example is that it also serves to show that, in an ordered regular semigroup in which every element $x$ has a biggest inverse $x^{0}$, the mapping $x \mapsto x^{00}$ is not in general a closure; nor is $x \mapsto x^{* *}$ in a principally ordered regular semigroup.
\end{abstract}

1991 Mathematics subject classification: 20M17, 06 F05.

The notion of an inverse transversal of a regular semigroup $S$ has been deeply investigated $[1,5,6,8,9,10,11,13]$. Basically, this consists of an inverse subsemigroup $T$ with the property that $|T \cap V(x)|=1$ for every $x \in S$ where as usual $V(x)$ denotes the set of inverses of $x$. We write the unique element of $T \cap V(x)$ as $x^{0}$, and $T$ as $S^{0}=\left\{x^{0}: x \in S\right\}$. If $E\left(S^{0}\right)$ is the semilattice of idempotents of $S^{0}$ then the inverse transversal $S^{0}$ is said to be multiplicative if $x^{0} x y y^{0} \in E\left(S^{0}\right)$ for all $x, y \in S$; and weakly multiplicative if $\left(x^{0} x y y^{0}\right)^{0} \in E\left(S^{0}\right)$ for all $x, y \in S$. The complicated structure of regular semigroups with inverse transversals of these types has been determined by Saito [13].

Related to an inverse transversal $S^{0}$ are the subsets $l=\left\{e \in S: e=e e^{0}\right\}$ and $\Lambda=$ $\left\{f \in S: f=f^{0} f\right\}$. These are sub-bands of $S$ [14]. As shown by Saito [8], $S^{0}$ is weakly multiplicative if and only if $I \Lambda=\{e f: e \in I, f \in \Lambda\}$ is the idempotent-generated subsemigroup of $S$ with inverse transversal $E\left(S^{0}\right)$. Whenever $S^{0}$ is a quasi-ideal of $S$, in the sense that $S^{0} S S^{0} \subseteq S^{0}$, I [resp. $\Lambda$ ] is a left [resp. right] normal band with semilattice transversal $E\left(S^{0}\right)$.

As observed by Saito [13], we have the following implications concerning an inverse transversal $S^{0}$ :

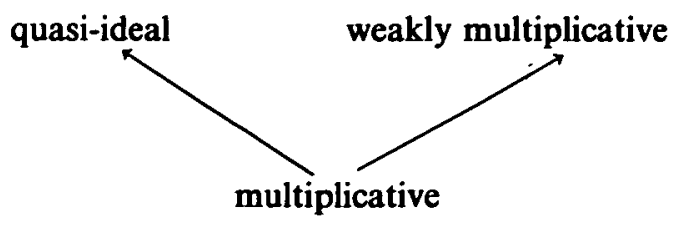

*NATO Collaborative Research Grant 910765 is gratefully acknowledged.

†Support from the Department of Mathematics, New University of Lisbon is gratefully acknowledged. 
Examples of multiplicative and quasi-ideal inverse transversals are to be found for example amongst the elementary rectangular bands of inverse semigroups [7] and regular semigroups whose idempotents form a four-spiral [3]. Here our objective is to describe an example of a weakly multiplicative inverse transversal that is neither multiplicative nor a quasi-ideal. The semigroup in question in surprisingly simple in that it consists of $2 \times 2$ matrices over a boolean algebra.

Before proceeding to this, however, we shall establish two results that are complementary to the following result of Saito [12].

Theorem 1. Let $S$ be a regular semigroup with an inverse transversal $S^{0}$. Then $S^{0}$ is multiplicative if and only if $S^{0}$ is a quasi-ideal of $S$ and $e^{0} \in E\left(S^{0}\right)$ for every $e \in E(S)$.

Theorem 2. Let $S$ be a regular semigroup with an inverse transversal $S^{0}$. Then the following statements are equivalent:

(1) $S^{0}$ is multiplicative;

(2) $S^{0}$ is weakly multiplicative and $\Lambda \mathrm{I} \subseteq S^{0}$.

Proof. (1) $\Rightarrow(2)$ : If $S^{0}$ is multiplicative then $x^{0} x y y^{0} \in E\left(S^{0}\right)$ for all $x, y \in S$. Therefore if $f \in \Lambda$ and $e \in I$ then $f e=f^{0} f e e^{0} \in E\left(S^{0}\right)$ whence $\Lambda \mathrm{I} \subseteq S^{0}$.

$(2) \Rightarrow(1)$ : Conversely, suppose that (2) holds. Then by [13, Lemma 2.3(6)] we have $(f e)^{00} \in E\left(S^{0}\right)$ for all $f \in \Lambda$ and $e \in \mathrm{I}$. But, by (2), we have $f e \in S^{0}$. Consequently $f e=(f e)^{00} \in E\left(S^{0}\right)$. Since $x^{0} x \in \Lambda$ and $y y^{0} \in I$ for all $x, y \in S$, it follows that $x^{0} x y y^{0} \in E\left(S^{0}\right)$, so $S^{0}$ is multiplicative.

In fact we have the following connection between the properties of being multiplicative, weakly multiplicative, and a quasi-ideal.

Theorem 3. An inverse transversal is multiplicative if and only if it is weakly multiplicative and a quasi-ideal.

Proof. The conditions are clearly necessary. Suppose conversely that $S^{0}$ is weakly multiplicative and a quasi-ideal. Then by $[13$, Lemma $2.3(7)]$ we have $(f e)^{00}=f e$ for all $f \in \Lambda$ and all $e \in I$, so $f e \in S^{0}$ and therefore, by Theorem, $2 S^{0}$ is multiplicative.

We now proceed to describe an example of an inverse transversal that is weakly multiplicative without being multiplicative. For this purpose, let $B$ be a boolean algebra and consider the set $B_{2}=M_{2 \times 2}(B)$ of $2 \times 2$ matrices with entries in $B$. It is well known that $\left(B_{2}, \leqq\right)$ is an ordered semigroup where $\leqq$ is given by

$$
\left[x_{i j}\right] \leqq\left[y_{i j}\right] \Leftrightarrow(\forall i, j) x_{i j} \leqq y_{i j}
$$

As shown by Luce [4], $B_{2}$ is in fact a residuated semigroup in which residuals are given 
by $X \cdot \cdot Y=\left(Y^{\prime} X^{\prime}\right)^{\prime}$ and $X \cdot . Y=\left(X^{\prime} Y^{\prime}\right)^{\prime}$. Because of this, $B_{2}$ is principally ordered [2] in the sense that for all $X \in B_{2}$ there exists

$$
X^{*}=\max \left\{Y \in B_{2}: X Y X \leqq X\right\} ;
$$

in fact we clearly have

$$
X^{*}=(X \cdot . X) \cdot X=(X \cdot X) \cdot X
$$

For convenience in what follows, we shall make use of the notation $x y, x+y, x^{\prime}$ to denote the operations in $B$. A simple calculation shows that if $X=\left[\begin{array}{ll}a & b \\ c & d\end{array}\right] \in B_{2}$, then

$$
X^{*}=\left[\begin{array}{ll}
b^{\prime}+c^{\prime}+d & a^{\prime}+b+d^{\prime} \\
a^{\prime}+c+d^{\prime} & a+b^{\prime}+c^{\prime}
\end{array}\right]
$$

We would warn the reader that we shall minimise most of the proofs that follow since they involve lengthy but elementary manipulations with these operations, using standard reductions such as the identities $x+x^{\prime} y=x+y$ and $(x+y) x^{\prime}=y x^{\prime}$, and the property $x y \leqq z \Leftrightarrow x \leqq z+y^{\prime}$.

Our first observation about $B_{2}$ is the following important fact.

Theorem 4. $B_{2}$ is regular.

Proof. Given $X=\left[\begin{array}{ll}a & b \\ c & d\end{array}\right] \in B_{2}$, consider the matrix

$$
X_{M}=\left[\begin{array}{ll}
a d+(b c)^{\prime} & b c+(a d)^{\prime} \\
b c+(a d)^{\prime} & a d+(b c)^{\prime}
\end{array}\right]
$$

A routine calculation reveals the identity $X X_{M} X=X$.

Corollary. $\left(\forall X \in B_{2}\right) X X^{*} X=X$.

Theorem 5. The set of idempotent of $B_{2}$ is

$$
E\left(B_{2}\right)=\left\{\left[\begin{array}{ll}
a & b \\
c & d
\end{array}\right]: b+c \leqq a+d, b c \leqq a d\right\}
$$

Proof. Again this follows by a routine calculation.

Theorem 6. Every element of $B_{2}$ has a biggest inverse.

Proof. Given $X=\left[\begin{array}{ll}a & b \\ c & d\end{array}\right] \in B_{2}$, consider the matrix 


$$
X^{0}=X^{*} X X^{*}=\left[\begin{array}{ll}
b^{\prime}(a+c)+c^{\prime}(a+b)+d & a^{\prime}(c+d)+d^{\prime}(a+c)+b \\
a^{\prime}(b+d)+d^{\prime}(a+b)+c & b^{\prime}(c+d)+c^{\prime}(b+d)+a
\end{array}\right]
$$

Since $B_{2}$ is principally ordered, it follows as in [2] that $X^{0}$ is the biggest inverse of $X$.

By standard properties of biggest inverses in ordered regular semigroups we have $X^{0}=X^{000}$ and $X \leqq X^{00}$. In what follows we shall write

$$
B_{2}^{0}=\left\{X^{0}: X \in B_{2}\right\}=\left\{X \in B_{2}: X=X^{00}\right\}
$$

Theorem 7. $X \in B_{2}^{0}$ if and only if the row and column sums of $X$ are equal.

Proof. If $X=\left[\begin{array}{ll}a & b \\ c & d\end{array}\right] \in B_{2}^{0}$ then using Theorem 6 we can see that

$$
X=X^{00}=\left[\begin{array}{ll}
b^{\prime}(c+d)+c^{\prime}(b+d)+a & a^{\prime}(c+d)+d^{\prime}(a+c)+b \\
a^{\prime}(b+d)+d^{\prime}(a+b)+c & b^{\prime}(a+c)+c^{\prime}(a+b)+d
\end{array}\right]
$$

which is a matrix whose row and column sums are equal to $a+b+c+d$. Then

Conversely, suppose that $Y=\left[\begin{array}{ll}x & y \\ z & w\end{array}\right] \in B_{2}$ is such that $x+y=z+w=x+z=y+w$.

$$
\begin{aligned}
Y^{00} & =\left[\begin{array}{ll}
y^{\prime}(z+w)+z^{\prime}(y+w)+x & x^{\prime}(z+w)+w^{\prime}(x+z)+y \\
x^{\prime}(y+w)+w^{\prime}(x+y)+z & y^{\prime}(x+z)+z^{\prime}(x+y)+w
\end{array}\right] \\
& =\left[\begin{array}{ll}
y^{\prime}(x+y)+z^{\prime}(x+z)+x & x^{\prime}(x+y)+w^{\prime}(y+w)+y \\
x^{\prime}(x+z)+w^{\prime}(z+w)+z & y^{\prime}(y+w)+z^{\prime}(z+w)+w
\end{array}\right] \\
& =\left[\begin{array}{ll}
x & y \\
z & w
\end{array}\right] \\
& =Y
\end{aligned}
$$

Corollary 1. $B_{2}^{0}$ is a subsemigroup of $B_{2}$.

Corollary 2. The idempotents of $B_{2}^{0}$ are the matrices of the form $\left[\begin{array}{ll}a & b \\ c & a\end{array}\right]$ with $b, c \leqq a$.

Proof. This follows from Theorem 5 and Theorem 7.

Theorem 8. $B_{2}^{0}$ is an inverse transversal of $B_{2}$.

Proof. The idempotents of $B_{2}^{0}$, as described in Corollary 2 of Theorem 7, are easily 
shown to commute. Since $X^{0} \in B_{2}^{0} \cap V(X)$ for every $X \in B_{2}$ it follows that $B_{2}^{0}$ is a regular semigroup whose idempotents commute, whence it is an inverse semigroup.

We now show that $\left|B_{2}^{0} \cap V(X)\right|=1$ for every $X \in B_{2}$. For this purpose we use the fact that if $X=\left[\begin{array}{ll}a & b \\ c & d\end{array}\right] \in B_{2}^{0}$ then $X^{-1}=X^{0}=\left[\begin{array}{ll}d & b \\ c & a\end{array}\right]$, so that in $B_{2}^{0}$ we have the property

$$
X \leqq Y \Rightarrow X^{0} \leqq Y^{0} \text {. }
$$

Suppose then that $Y \in B_{2}^{0} \cap V(X)$. Since $Y \leqq X^{0}$ we have $Y^{0} \leqq X^{00}$ and therefore

$$
Y=Y Y^{0} Y \leqq Y X^{00} Y=Y X Y X^{00} Y X Y \leqq Y X X^{0} X^{00} X^{0} X Y=Y X Y=Y
$$

whence $Y=Y X^{00} Y$. Also,

$$
\begin{aligned}
X^{00}=X^{00} X^{0} X X^{0} X^{00} & =X^{00} X^{0} X Y X X^{0} X^{00} \\
& \leqq X^{00} X^{0} X^{00} Y X^{00} X^{0} X^{00} \\
& =X^{00} Y X^{00} \\
& \leqq X^{00} X^{0} X^{00} \\
& =X^{00}
\end{aligned}
$$

whence $X^{00}=X^{00} Y X^{00}$. Thus $X^{00} \in V(Y)$ and so $X^{00} \leqq Y^{0}$. But $Y^{0} \leqq X^{00}$ so $Y^{0}=X^{00}$ and consequently $Y=Y^{00}=X^{0}$. Hence we have $B_{2}^{0} \cap V(X)=\left\{X^{0}\right\}$.

Theorem 9. $\quad B_{2}^{0}$ is neither multiplicative nor a quasi-ideal.

Proof. By way of obtaining contradiction, suppose that $B_{2}^{0}$ is multiplicative. Then we have $X^{0} X Y Y^{0} \in E\left(B_{2}^{0}\right)$ for all $X, Y \in B_{2}$. Taking $Y$ to be the identity matrix in this, we obtain $X^{0} X \in E\left(B_{2}^{0}\right)$. But for $X=\left[\begin{array}{ll}a & b \\ c & d\end{array}\right]$ we have

$$
X^{0} X=\left[\begin{array}{cc}
a+c & b c^{\prime}+b d+a^{\prime} d \\
a d^{\prime}+a c+b^{\prime} c & b+d
\end{array}\right]
$$

and by Corollary 2 of Theorem 7 this need not belong to $E\left(B_{2}^{0}\right)$, contrary to the hypothesis. Hence $B_{2}^{0}$ is not multiplicative.

That $B_{2}^{0}$ is not a quasi-ideal follows immediately from the identity

$$
\left[\begin{array}{ll}
0 & 1 \\
1 & 0
\end{array}\right]\left[\begin{array}{ll}
a & b \\
c & d
\end{array}\right]\left[\begin{array}{ll}
0 & 1 \\
1 & 0
\end{array}\right]=\left[\begin{array}{ll}
d & c \\
b & a
\end{array}\right]
$$


and Theorem 7.

Our objective now is to prove that the inverse transversal $B_{2}^{0}$ is weakly multiplicative. By a result of Saito mentioned above, it suffices to prove that $\left\langle E\left(B_{2}\right)\right\rangle=I \Lambda$ and has inverse transversal $E\left(B_{2}^{0}\right)$. For this purpose it is necessary to identify $\left\langle E\left(B_{2}\right)\right\rangle$.

Theorem 10. $\left\langle E\left(B_{2}\right)\right\rangle=\left\{\left[\begin{array}{ll}a & b \\ c & d\end{array}\right]: b c \leqq a d\right\}$.

Proof. Denote by $S$ the set of matrices in question. Then $S$ is a subsemigroup of $B_{2}$; for if $X=\left[\begin{array}{ll}a & b \\ c & d\end{array}\right]$ and $Y=\left[\begin{array}{ll}x & y \\ z & w\end{array}\right]$ belong to $S$ then

$$
\begin{aligned}
(a y+b w)(c x+d z) & =a c x y+a d y z+b c w x+b d w z \\
& \leqq a c x y+a d w x+a d w x+b d w z \\
& \leqq a c x y+a d w x+b d w z+b c y z \\
& =(a x+b z)(c y+d w)
\end{aligned}
$$

and therefore $X Y \in S$. It follows immediately from Theorem 5 that $\left\langle E\left(B_{2}\right)\right\rangle \subseteq S$.

To establish the reverse inclusion, observe that if $X \in S$ and if $X_{M}$ is as in Theorem 4, then $X X_{M}$ and $X_{M} X$ are idempotents, as is also $X_{M}$ by Theorem 5. Consequently we have

$$
X=X X_{M} X=X X_{M} X_{M} X \in\left\langle E\left(B_{2}\right)\right\rangle
$$

We can now establish the main result.

Theorem 11. $B_{2}^{0}$ is weakly multiplicative.

Proof. A routine calculation gives

$$
\mathbf{I}=\left\{X \in B_{2}: X=X X^{0}\right\}=\left\{\left[\begin{array}{ll}
a & b \\
c & d
\end{array}\right]: a+d=b+d=a+c, b \leqq a, c \leqq d\right\}
$$

and similarly

$$
\Lambda=\left\{X \in B_{2}: X=X^{0} X\right\}=\left\{\left[\begin{array}{ll}
a & b \\
c & d
\end{array}\right]: a+d=c+d=a+b, c \leqq a, b \leqq d\right\} .
$$

As $I$ and $\Lambda$ are both sets of idempotents, we have $I \Lambda \subseteq\left\langle E\left(B_{2}\right)\right\rangle$. To establish the reverse inclusion note that, as observed in the proof of Theorem 10 , every $X \in\left\langle E\left(B_{2}\right)\right\rangle$ is such that $X=X X_{M} \cdot X_{M} X$. But for $X=\left[\begin{array}{ll}a & b \\ c & d\end{array}\right] \epsilon\left\langle E\left(B_{2}\right)\right\rangle$ we have, by Theorems 4 and 10 , 
that

$$
X_{M}=\left[\begin{array}{cc}
1 & b c+(a d)^{\prime} \\
b c+(a d)^{\prime} & 1
\end{array}\right]
$$

from which another routine caluclation shows that

$$
X X_{M}=\left[\begin{array}{cc}
a+b & a d^{\prime}+b \\
c+a^{\prime} d & c+d
\end{array}\right] \in \mathbf{I}
$$

and similarly $X_{M} X \in \Lambda$. It follows that $\left\langle E\left(B_{2}\right)\right\rangle \subseteq \mathrm{I} \Lambda$ and therefore $\left\langle E\left(B_{2}\right)\right\rangle=\mathrm{I} \Lambda$.

To prove that $E\left(B_{2}^{0}\right)$ is an inverse transversal of $\left\langle E\left(B_{2}\right)\right\rangle$, we observe that

$$
E\left(B_{2}^{0}\right) \cap V(X) \subseteq B_{2}^{0} \cap V(X)=\left\{X^{0}\right\},
$$

so it suffices to prove that for every $X \in\left\langle E\left(B_{2}\right)\right\rangle$ we have $X^{0} \in E\left(B_{2}^{0}\right)$. For this purpose, suppose that $X=\left[\begin{array}{ll}a & b \\ c & d\end{array}\right] \in\left\langle E\left(B_{2}\right)\right\rangle$ and let $X^{0}=\left[\begin{array}{ll}x & y \\ z & w\end{array}\right]$. By Corollary 2 of Theorem 7 we have to show that $x=w$ and that $y, z \leqq x$. Now by Theorem 6 we have

$$
\begin{aligned}
& x=b^{\prime}(a+c)+c^{\prime}(a+b)+d=(b c)^{\prime}(a+b+c)+d, \\
& w=b^{\prime}(c+d)+c^{\prime}(b+d)+a=(b c)^{\prime}(b+c+d)+a .
\end{aligned}
$$

Since, by Theorem $10, b c \leqq a d$ we have

$$
\begin{aligned}
a+b+c+d & \geqq(b c)^{\prime}(a+b+c)+d=x \\
& \geqq(a d)^{\prime}(a+b+c)+d \\
& =(a d)^{\prime}(a+b+c)+d+a d(a+b+c) \\
& =a+b+c+d,
\end{aligned}
$$

whence $x=a+b+c+d$; and similarly $w=a+b+c+d$. It now follows by Theorem 7 that

whence $y, z \leqq x$.

$$
x+y=x+z=a+b+c+d=x
$$

We close with the following interesting property of the semigroup $B_{2}$. As we have seen above, $B_{2}$ is an ordered regular semigroup in which every element $X$ has a biggest inverse $X^{0}$. Since $X^{0}=X^{000}$ and $X \leqq X^{00}$, the mapping $\theta: X \mapsto X^{00}$ is such that $\theta^{2}=\theta \geqq i d$ and therefore satisfies the axioms for a closure except perhaps isotonicity. In fact, in this case the mapping $\theta$ is not isotone on $B_{2}$. To see this, consider the matrices 


$$
M_{1}=\left[\begin{array}{ll}
0 & 1 \\
0 & 0
\end{array}\right], \quad M_{2}=\left[\begin{array}{ll}
0 & 1 \\
1 & 0
\end{array}\right]
$$

For these matrices, we have

$$
M_{1}^{00}=\left[\begin{array}{ll}
1 & 1 \\
1 & 1
\end{array}\right], \quad M_{2}^{00}=\left[\begin{array}{ll}
0 & 1 \\
1 & 0
\end{array}\right] .
$$

Clearly, $M_{1} \leqq M_{2}$ but $M_{1}^{00} \leq M_{2}^{00}$.

In contrast, note that $\theta$ is a closure when restricted to $B_{2}^{0}$. This follows from the fact, observed in the proof of Theorem 8, that the mapping $\phi: X \mapsto X^{0}$ is isotone on $B_{2}^{0}$.

In general, for an ordered regular semigroup with biggest inverses, necessary and sufficient conditions for $x \mapsto x^{00}$ to be a closure are unknown.

A similar observation can be made concerning the mapping $\zeta: X \mapsto X^{* *}$. Here the subset

$$
B_{2}^{*}=\left\{X^{*}: X \in B_{2}\right\}=\left\{X \in B_{2}: X=X^{* *}\right\}
$$

is an inverse subsemigroup of $B_{2}^{0}$. The elements of $B_{2}^{*}$ are those $X$ whose row and column sums are equal to 1 , and the idempotents of $B_{2}^{*}$ are the matrices of the form

$$
\left[\begin{array}{ll}
1 & b \\
c & 1
\end{array}\right]
$$

For $X=\left[\begin{array}{ll}a & b \\ c & d\end{array}\right] \in B_{2}$ it is readily seen that

$$
X^{* *}=\left[\begin{array}{ll}
a+b^{\prime}+c^{\prime} & a^{\prime}+b+d^{\prime} \\
a^{\prime}+c+d^{\prime} & b^{\prime}+c^{\prime}+d
\end{array}\right]
$$

so that, for $M_{1}$ and $M_{2}$ above, we have

$$
M_{1}^{* *}=\left[\begin{array}{ll}
1 & 1 \\
1 & 1
\end{array}\right], \quad M_{2}^{* *}=\left[\begin{array}{ll}
0 & 1 \\
1 & 0
\end{array}\right]
$$

whence $M_{1}^{* *} \$ M_{2}^{* *}$.

In general, for a principally ordered regular semigroup, necessary and sufficient conditions for $x \mapsto x^{* *}$ to be a closure are unknown.

\section{REFERENCES}

1. T. S. Blyth and R. MCFADDEN, Regular semigroups with a multiplicative inverse transversal, Proc. Roy. Soc. Edinburgh 92A (1982), 253-270. 
2. T. S. Blyth and G. A. Pinto, Principally ordered regular semigroups, Glasgow Math. J. 32 (1990), 349-364.

3. K. ByleEn, Regular four-spiral semigroups, idempotent semigroups and the Rees construction, Semigroup Forum 22 (1981), 97-100.

4. R. D. LuCE, A note on boolean matrix theory, Proc. Amer. Math. Soc. 3 (1952), 382-388.

5. D. B. MCAluster and R. MCFADDEN, Regular semigroups with inverse transversals, Quart. J. Math. Oxford 34 (1983), 459-474.

6. D. B. McAlister and R. McFadden, Semigroups with inverse transversals as matrix semigroups, Quart. J. Math. Oxford 35 (1984), 455-474.

7. F. Pastijn, Rectangular bands of inverse semigroups. Simon Stevin 56 (1982), 1-97.

8. Tatsuhiko Sarto, Regular semigroups with a weakly multiplicative inverse transversal, in Proc. 8th Symposium on Semigroups (Shimane University, 1985), 22-25.

9. Tatsuhiko Sarto, Structure of regular semigroups with a quasi-ideal inverse transversal, Semigroup Forum 31 (1985), 305-309.

10. TAtsuhixo SAIto, Relationship between the inverse transversals of a regular semigroup, Semigroup Forum 33 (1986), 245-250.

11. Tatsuhiko Saito, A note on regular semigroups with inverse transversals, Semigroup Forum 33 (1986), 149-152.

12. TAтsuhiko SAIto, Naturally ordered regular semigroups with maximum inverses, Proc. Edinburgh Math. Soc. 32 (1989), 33-39.

13. Tatsuniko SaIto, Construction of regular semigroups with inverse transversals, Proc. Edinburgh Math. Soc. 32 (1989), 41-51.

14. XILIN TANG, Regular semigroups with inverse transversals (preprint).

Mathematical Institute

UNIVERSITY OF ST ANDREWS

SCOTLAND
Departamento de Matemática UnIVERSIDADE Nova DE LISBOA Portugal 